\title{
Integrated nanobiosensor technology for biomedical application
}

This article was published in the following Dove Press journal:

Nanobiosensors in Disease Diagnosis

16 February 2012

Number of times this article has been viewed

\author{
Chulhee Choi \\ 'Department of Bio and Brain \\ Engineering, ${ }^{2} \mathrm{Graduate}$ School \\ of Medical Science and Engineering, \\ ${ }^{3} \mathrm{KI}$ for the BioCentury ${ }^{4}$ Optical \\ Bioimaging Center, KAIST, Daejeon, \\ Republic of Korea
}

\begin{abstract}
Advances in nanotechnology have led to the development of nanoscale biosensors that have exquisite sensitivity and versatility. The biomedical application of nanobiosensors is wide; moreover, the future impact of nanobiosensor systems for point-of-care diagnostics will be unmatched. The ultimate goal of nanobiosensors is to detect any biochemical and biophysical signal associated with a specific disease at the level of a single molecule or cell. This technology will revolutionize conventional medical practices by enabling early diagnosis of chronic debilitating diseases, ultrasensitive detection of pathogens, and long-term monitoring of patients using biocompatible integrated medical instrumentation. In the present commentary, current state-of-the art principles of nanobiosensor systems and integration into lab-on-a-chip devices are discussed along with future perspectives.
\end{abstract}

Keywords: nanotechnology, biosensor, diagnostics, point-of-care, lab-on-a-chip, microfluidics

\section{Nanobiosensors in biomedical application}

Nanobiosensors are analytical devices that utilize nanoscale detector components to identify minute biological elements with enhanced sensitivity. "Nanoscale" commonly refers to the ranges below the wavelength of visible light (usually shorter than $100 \mathrm{~nm}$ ). Because of the quantum effects, physicochemical behaviors at this scale are quite unique compared to those at conventional macroscopic scales. Besides nanoscale-specific physical properties, extremely large surface-to-volume ratios achieved by various nanoscale architectures dramatically increase the sensitivity of biosensors. Different nanoscale architectures such as nanowires, nanotubes, and nanofibers have unique advantages and limitations, which are discussed in a review by Bellan et al. ${ }^{1}$

The ability to detect disease-associated biomolecules, such as disease-specific metabolites, nucleic acids, proteins, pathogens, and cells such as circulating tumor cells, is essential not only for disease diagnosis in the clinical setting but also for biomedical research involving drug discovery and development. Nanotechnology, with its enhanced sensitivity and reduced instrumentation size, will rapidly improve our current biodiagnostic capacity with respect to specificity, speed, and cost. Reduction in sensor size provides great versatility for incorporation into multiplexed, transportable, portable, wearable, and even implantable medical devices. The integration of nanoscale ultrasensitive biosensors with other medical instruments will open the door to emerging medical fields, including point-of-care (POC) diagnostics and ubiquitous healthcare systems. ${ }^{2}$
Correspondence: Chulhee Choi

Department of Bio and Brain Engineering, KAIST, 29I Daehak-ro, Yuseong-gu,

Daejeon 30I-705, Republic of Korea

Tel +82 423504321

Fax +8242350 438।

Email cchoi@kaist.ac.kr 


\section{Nanobiosensor systems}

Biosensors are generally composed of three elements: the biologically sensitive element, the transducer or detector element, and the biosensor reader device. In this section, common configurations of state-of-the-art nanobiosensors and the integration into affordable instrumentation are discussed, with a focus on microfluidics and implantable electronic devices.

\section{Detection principles used for nanobiosensors}

Biosensor recognition elements often mimic naturally occurring biomolecules isolated from living systems. Thus, recent methods of molecular recognition employ modern biotechnology and molecularly imprinted polymer engineering (for a detailed discussion, please refer to a review by Chambers et al). ${ }^{3}$ The transducer converts the signals produced by the physicochemical process of the biologically sensed elements into measurable and quantifiable electrical signals.

\section{Molecular recognition}

Molecular recognition is a physiologic mechanism that often involves transmembrane cell surface receptors that are specific for a variety of soluble ligands such as proteins, toxins, drugs, and pathogens. Genetic engineering technology has enabled not only reliable industry-level production of macromolecular receptors but also affinity optimization of receptor proteins using sequence engineering. Direct monitoring of receptor-ligand interactions has also become possible via recent advances in ultrasensitive detection methods, such as surface plasmon resonance and surfaceenhanced Raman scattering. Functional proteins, such as catalytic enzymes, represent another attractive recognition mechanism. Catalytic enzymatic reactions involve various physicochemical components such as protons, electrons, light, and heat, which can be readily transformed into measurable electrical signals. Another functional protein, the antibody, has been applied in molecular recognition due to the high sensitivity, antigen specificity, and well-established monoclonal antibody technology. Moreover, lectin family proteins are also excellent biorecognition molecules owing to their high affinity for a variety of polysaccharide ligands. ${ }^{4}$ Recently, genetically encoded biosensors, such as translocation-based protein probes and ratiometric-based fluorescence proteins, are used for real-time biochemistry of living cells and whole organisms. ${ }^{5}$

Nucleic acids, such as oligonucleotides and aptamers, are available for molecular recognition due to their high specificity for complimentary oligonucleotides and polypeptides. One advantage of the nucleic acid-based recognition mechanism is the feasibility for high density integration, as seen in the array systems. Synthetic nucleic acid analogs, such as peptide nucleic acids, are attractive candidates for molecular sensors due to superior hybridization affinity and improved chemical and enzymatic stability. ${ }^{6}$

Cell- or tissue-based biosensors are also being developed as potential sensing elements, owing to their superior sensitivity for specific ligands, to detect the physiologic status of receptors and to sense multiplexed complexes. ${ }^{7}$ Examples of the ultrasensitivity of cell-based sensors are found in the olfactory neurons, retinal neurons, and T lymphocytes that are capable of responding to a single odorant molecule, a photon, or an antigen molecule, respectively. Various cellular responses are also readily converted into measurable electrical signals by various conventional transducing methods. However, low reproducibility limits the wide application of cell-based sensors. Cell - and tissuebased sensors have been designed for their potential in pharmacokinetic and toxicological profiling of newly developed drugs. ${ }^{8}$ Organisms such as bacteria or viruses have recently proven useful for the detection of target analytes such as explosives, proteins, bacteria, viruses, spores, and toxins with high selectivity and sensitivity. ${ }^{9}$ For example, a thin film sensor for the detection of volatile organic compounds was successfully fabricated using the tobacco mosaic virus. ${ }^{10}$

\section{Signal transducing principles}

Basic biosensor systems include electrochemical or optical transducers, and mechanical, piezoelectrical, electrical, calorimetric, and acoustic transduction mechanisms have also been applied. In the nanoscale range, the sensitivity of signal detection is drastically improved due to nanoscale-specific physical properties. ${ }^{11}$ For example, relatively weak optical signals such as surface plasmon resonance and surfaceenhanced Raman scattering can be applied as highly sensitive and specific biosensors in conjunction with various nanoarchitectures. ${ }^{12}$ Besides superior sensitivity, the miniaturized sensors may provide additional benefits such as parallel operation, portability, and low power consumption.

The calorimetric sensing method has recently been highlighted as a novel biosensor with several advantages over other methods, including the ubiquity of heat, label-free sensing, and noninvasive passive sensing (for a detailed discussion, please refer to a review by Lee et al).${ }^{13}$ Miniaturized calorimetric systems have great potential to be used as unique 
and powerful biosensors, as the problem of low sensitivity due to heat loss has been addressed by the recent development of meticulous microfluidic configurations. Because of its noninvasiveness and passive sensing, calorimetric measurement can provide nondamaging metabolic measurements of live cells and tissues. In addition, long-term monitoring of biochemical reactions or interactions in a cell-on-a-chip or animal-on-a-chip format can be monitored calorimetrically.

\section{Integration of nanobiosensor systems}

Rapid and convenient detection is essential for portable clinical monitoring systems, such as handheld POC testing devices. In addition, miniaturization and conformal contact on curvilinear surfaces are necessary to allow the implantable microsized electronic system to function in real-time healthcare applications in vivo. The advent of nanofabrication technology has allowed the miniaturization of conventional benchtop instruments, resulting in devices such as the nano/ microelectromechanical systems, microfluidic embedded sensor systems, and integrated systems such as the micro Total Analysis System. ${ }^{14}$

\section{Lab-on-a-chip configuration}

Microfluidics is an essential technology utilized in labon-a-chip diagnostic systems. Microfluidics technology avoids some of the main challenges in traditional assays by integrating sensing and sample pretreatment modules, reducing valuable reagent consumption, increasing the overall efficiency of the assays, and avoiding crosscontamination. ${ }^{15}$ As a result, fast, selective, and portable microfluidic devices enhance POC diagnostics.

\section{Biocompatible medical devices}

Generated via nanofabrication technologies, flexible and implantable biomedical devices are now available that use in vivo sensing electrodes, such as the nano/microelectromechanical systems and the dry transfer printing method. Advances in nanofabrication technologies overcome the mechanical limits of brittle inorganic-based devices, and offer flexible and stretchable electronics for innovative applications. As a pioneer in flexible electronic devices, laser emission diode-based therapeutic and diagnostic systems have been successfully incorporated into flexible systems, and have been proven effective in biomedical areas such as wound healing, optogenetics, photodynamic therapy, and biomolecular sensing (for a perspective discussion, please refer to a review by Koo et al). ${ }^{16}$
Nanobiosensors in Disease Diagnosis: an open-access journal that integrates multidisciplinary research and development in medical diagnostics

Population growth and the increasing number of people suffering from chronic debilitating illnesses are the main causes for the growing biosensor market in medical diagnostics. As more people become aware of health-related issues, the demand for biosensors in disease diagnosis will grow rapidly, especially for real-time POC testing and long-term monitoring. As we have glimpsed how the array of technologies from different disciplines are incorporated in this unprecedented medical progress, the journal Nanobiosensors in Disease Diagnosis will be a platform that integrates a range of academic and industrial advances that can lead to the next generation of personalized and ubiquitous healthcare systems and achieve extraordinary advances in modern medical sciences. This new open-access journal makes cutting-edge knowledge freely available to researchers worldwide, and this is particularly important for integrating multidisciplinary research. We sincerely welcome contributions from all areas of science and technology related to integrated nanobiosensor systems (including but not confined to biotechnology, medicine, pharmacology, microbiology, immunology, pharmacy, nanotechnology, microelectromechanical systems, microfluidics, biophotonics, bioelectronics, molecular modeling, material sciences, physics, and chemistry). The editorial board of Nanobiosensors in Disease Diagnosis is made up of an expert group of investigators and researchers from a wide range of disciplines and regions. Finally, Nanobiosensors in Disease Diagnosis has one of the fastest turnaround times of any medical journal in the world.

\section{Acknowledgments}

This study is supported by the Korea Science and Engineering Foundation (KOSEF) grant, funded by the Korean government (MEST No 2010-00300001).

\section{Disclosure}

The author reports no conflicts of interest in this work. The funders had no role in the study design, data collection and analysis, manuscript preparation, or the decision to publish.

\section{References}

1. Bellan LM, Wu D, Langer RS. Current trends in nanobiosensor technology. Wiley Interdiscip Rev Nanomed Nanobiotechnol. 2011;3(3): 229-246. 
2. Yager P, Domingo GJ, Gerdes J. Point-of-care diagnostics for global health. Annu Rev Biomed Eng. 2008;10:107-144.

3. Chambers JP, Arulanandam BP, Matta LL, Weis A, Valdes JJ. Biosensor recognition elements. Curr Issues Mol Biol. 2008;10(1-2):1-12.

4. Xie M, Hu J, Long YM, Zhang ZL, Xie HY, Pang DW. Lectin-modified trifunctional nanobiosensors for mapping cell surface glycoconjugates. Biosens Bioelectron. 2009;24(5):1311-1317.

5. Palmer AE, Qin Y, Park JG, McCombs JE. Design and application of genetically encoded biosensors. Trends Biotechnol. 2011;29(3): 144-152.

6. Singh RP, Oh BK, Choi JW. Application of peptide nucleic acid towards development of nanobiosensor arrays. Bioelectrochemistry. 2010;79(2):153-161.

7. Banerjee P, Bhunia AK. Mammalian cell-based biosensors for pathogens and toxins. Trends Biotechnol. 2009;27(3):179-188.

8. Xi B, Yu N, Wang X, Xu X, Abassi YA. The application of cell-based label-free technology in drug discovery. Biotechnol J. 2008;3(4): 484-495.
9. Mao C, Liu A, Cao B. Virus-based chemical and biological sensing. Angew Chem Int Ed Engl. 2009;48(37):6790-6810.

10. Bruckman MA, Liu J, Koley G, et al. Tobacco mosaic virus based thin film sensor for detection of volatile organic compounds. J Mater Chem. 2010;20(27):5715-5719.

11. Cullum BM, Vo-Dinh T. The development of optical nanosensors for biological measurements. Trends Biotechnol. 2000;18(9):388-393.

12. Erickson D, Mandal S, Yang A, Cordovez B. Nanobiosensors: optofluidic, electrical and mechanical approaches to biomolecular detection at the nanoscale. Microfluid Nanofluidics. 2008;4(1-2):33-52.

13. Lee W, Lee J, Koh J. Development and applications of chip calorimeters as novel biosensors. Nanobiosensors in Disease Diagnosis. In press.

14. Voldman J, Gray ML, Schmidt MA. Microfabrication in biology and medicine. Annu Rev Biomed Eng. 1999;1:401-425.

15. Haeberle S, Zengerle R. Microfluidic platforms for lab-on-a-chip applications. Lab Chip. 2007;7(9):1094-1110.

16. Koo M, Park SY, Lee KJ. Bio-integrated flexible inorganic LED. Nanobiosensors in Disease Diagnosis. In press.

\section{Publish your work in this journal}

Nanobiosensors in Disease Diagnosis is an international, peer-reviewed, open access journal publishing original research, reports, reviews and commentaries including but not confined to: Diagnosis of diseases including cancer, cardiovascular, infectious diseases; Molecular modelling in diagnosis; Enzyme and membrane technologies; and quantum dot fluorescence technologies for monitoring toxins and pathogens. The manuscript management system is completely online and includes a very quick and fair peer-review system, which is all easy to use. Visit http://www.dovepress.com/testimonials.php to read real quotes from published authors.

Submit your manuscript here: http://www.dovepress.com/nanobiosensors-in-disease-diagnosis-journal 